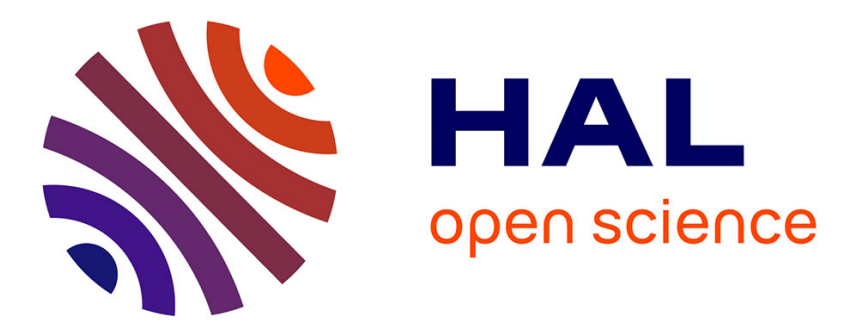

\title{
Efficient generation of surface plasmon by single-nanoslit illumination under highly oblique incidence
}

\author{
Bing Wang, Lionel Aigouy, E. Bourhis, J. Gierak, Jean-Paul Hugonin, \\ Philippe Lalanne
}

\section{- To cite this version:}

Bing Wang, Lionel Aigouy, E. Bourhis, J. Gierak, Jean-Paul Hugonin, et al.. Efficient generation of surface plasmon by single-nanoslit illumination under highly oblique incidence. Applied Physics Letters, 2009, 94 (1), pp.011114. 10.1063/1.3068747 . hal-00570593

HAL Id: hal-00570593

https://hal-iogs.archives-ouvertes.fr/hal-00570593

Submitted on 5 Apr 2012

HAL is a multi-disciplinary open access archive for the deposit and dissemination of scientific research documents, whether they are published or not. The documents may come from teaching and research institutions in France or abroad, or from public or private research centers.
L'archive ouverte pluridisciplinaire HAL, est destinée au dépôt et à la diffusion de documents scientifiques de niveau recherche, publiés ou non, émanant des établissements d'enseignement et de recherche français ou étrangers, des laboratoires publics ou privés. 


\section{$1 D$ Applied Physics \\ Letters \\ Efficient generation of surface plasmon by single-nanoslit illumination under highly oblique incidence}

B. Wang, L. Aigouy, E. Bourhis, J. Gierak, J. P. Hugonin et al.

Citation: Appl. Phys. Lett. 94, 011114 (2009); doi: 10.1063/1.3068747

View online: http://dx.doi.org/10.1063/1.3068747

View Table of Contents: http://apl.aip.org/resource/1/APPLAB/v94/i1

Published by the American Institute of Physics.

\section{Related Articles}

Optical coupling of surface plasmons between graphene sheets

Appl. Phys. Lett. 100, 131111 (2012)

Tuning asymmetry parameter of Fano resonance of spoof surface plasmons by modes coupling Appl. Phys. Lett. 100, 131110 (2012)

Resonant surface magnetoplasmons in two-dimensional magnetoplasmonic crystals excited in Faraday configuration

J. Appl. Phys. 111, 07A946 (2012)

Robust exciton-polariton effect in a $\mathrm{ZnO}$ whispering gallery microcavity at high temperature

Appl. Phys. Lett. 100, 101912 (2012)

Surface wave resonances supported on a square array of square metallic pillars

Appl. Phys. Lett. 100, 101107 (2012)

\section{Additional information on Appl. Phys. Lett.}

Journal Homepage: http://apl.aip.org/

Journal Information: http://apl.aip.org/about/about_the_journal

Top downloads: http://apl.aip.org/features/most_downloaded

Information for Authors: http://apl.aip.org/authors

\section{ADVERTISEMENT}

NEW!

iPeerReview

AIP's Newest App
Authors...

Reviewers...

Check the status of

submitted papers remotely!

ADPublishing 


\title{
Efficient generation of surface plasmon by single-nanoslit illumination under highly oblique incidence
}

\author{
B. Wang, ${ }^{1, a)}$ L. Aigouy, ${ }^{2, b)}$ E. Bourhis, ${ }^{3}$ J. Gierak, ${ }^{3}$ J. P. Hugonin, ${ }^{1}$ and P. Lalanne ${ }^{1}$ \\ ${ }^{1}$ Laboratoire Charles Fabry de l'Institut d'Optique, CNRS, Univ Paris-Sud, Campus Polytechnique, \\ RD 128, 91127 Palaiseau Cedex, France \\ ${ }^{2}$ Laboratoire Photons Et Matière, CNRS UPR 5, ESPCI, 10 rue Vauquelin, 75231 Paris Cedex 5, France \\ ${ }^{3}$ Laboratoire de Photonique et de Nanostructures, CNRS UPR 20, Route de Nozay, \\ 91460 Marcoussis, France
}

(Received 7 October 2008; accepted 11 December 2008; published online 9 January 2009)

Using scanning near-field optical microscopy, we investigate the ability of nanoslits in metallic films to launch surface plasmon polaritons (SPPs) under highly oblique incidence at $\lambda=975 \mathrm{~nm}$. The SPP generation efficiency is inferred by fitting the recorded near-field data with a simple analytical model. We find a remarkably large efficiency of $20 \%$ for the front side of the slit, which is in agreement with recent theoretical predictions relying on a fully vectoral electromagnetic formalism. An even larger efficiency is predicted experimentally (44\%) and theoretically (33\%) for the rear side. The present near-field analysis provides a direct approach to measure SPP generation efficiencies and may find applications for characterizing SPP devices. (c) 2009 American Institute of Physics. [DOI: 10.1063/1.3068747]

As the potential information carrier in future optical communication, surface plasmon polaritons (SPPs) have attracted much attention due to their remarkable ability for breaking the diffraction limit. ${ }^{1}$ Optical plasmonic devices operating with SPPs are expected to possess diminished sizes by comparison with those achieved with dielectric devices. So far a large diversity of active and passive optical nanodevices based on SPPs has been theoretically proposed and experimentally demonstrated. ${ }^{2}$ A key device is a SPP generator that may efficiently convert the exciting field (often light) into SPPs. Traditional SPP generators or antennas such as prisms and gratings seem too bulky to be integrated in large scale. With the development of nanotechnology, subwavelength structures offering small footprints such as slits or ridges on metal surfaces are currently investigated ${ }^{3-11}$ to generate SPPs. The SPP generation efficiency has been experimentally investigated by detecting SPPs in the near-field region $^{8}$ or by measuring their leakage in the far-field region. ${ }^{3,10}$ However, only normally incident light was considered in these previous works. As predicted in a recent theoretical study, ${ }^{2}$ highly oblique incidence may allow higher SPP generation efficiency.

In this letter, we present a near-field optical study of an isolated nanoslit aperture, illuminated at oblique incidence. The near-field measurement is performed in the reflection mode by recording the near field at the side illuminated by the incident light. We will show that the presence of the slit strongly modifies the standing wave usually observed on illuminated flat metallic surfaces. For TM polarization (magnetic field parallel to the slit), the near-field pattern contains two SPPs generated on each side of the aperture and a nonplasmonic field localized near the slit. Since these waves interfere with the specular incident and reflected fields, two oscillating patterns are observed on each side of the slit. From the near-field fringes, we can infer the SPP generation efficiency at the given incidence.

\footnotetext{
${ }^{a)}$ Electronic mail: bing.wang @institutoptique.fr.

${ }^{b)}$ Electronic mail: aigouy @optique.espci.fr.
}

The experimental configuration is represented in Fig. 1(a). The sample consists in a $280 \mathrm{~nm}$ large nanoslit [Fig. 1(b)] etched in a 100 -nm-thick gold film by a focused ionbeam apparatus. ${ }^{13}$ It is laterally illuminated by an intensity modulated laser diode $(\lambda=975 \mathrm{~nm})$, with an angle of incidence $\theta \approx 75^{\circ}$ from the surface normal [Fig. 1(a)]. At this incident angle, the beam impinging the metal surface is over $100 \mu \mathrm{m}$ in width, largely covering the entire slit. Thus the incident wave can be regarded as a plane wave in this case. The plane of incidence is perpendicular to the slit axis and the incident beam polarization is either in the plane of incidence (TM) or perpendicular to it (TE). The near-field probe is a fluorescent erbium/ytterbium codoped fluoride glass particle with a size close to $200 \mathrm{~nm} .{ }^{6}$ The particle absorbs the near-infrared photons and re-emits light in the visible by a nonlinear up-conversion process. For such a material, the measured fluorescence signal is proportional to the square of
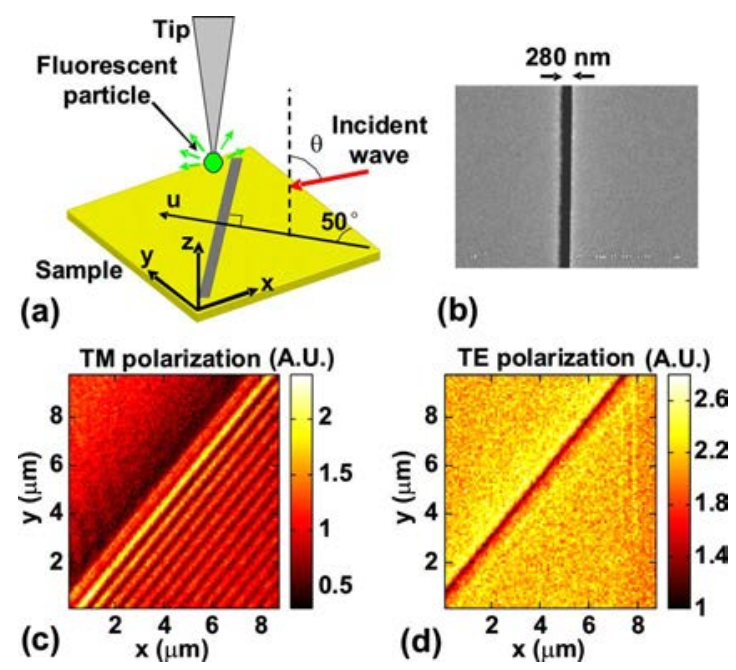

FIG. 1. (Color online) (a) Experimental configuration. The slit makes a $50^{\circ}$ angle with the $x$ axis. The plane of incidence is defined by the vertical $z$ - and $u$-directions. (b) Scanning electron microscope image of the slit. (c) Nearfield optical image for a TM-polarized incident illumination. (d) Same for TE. 


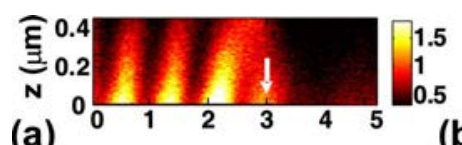

(a)

(b)
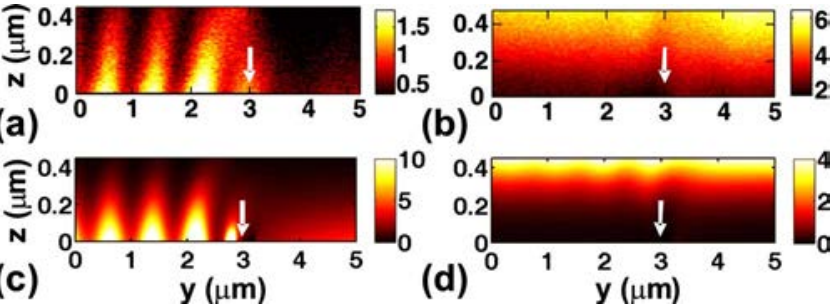

FIG. 2. (Color online) [(a) and (b)] Experimental measurement and [(c) and (d)] numerical simulation of the near-field distribution in a $y$ - $z$ plane above the slit aperture, for [(a) and (c)] TM or [(b) and (d)] TE polarizations. In (c) and (d) the computed data represent the square of the total electric-field intensity $|E|^{4}$ and the slit center is located at $y=3 \mu \mathrm{m}$. The white arrows indicate the position of the slit.

the intensity of the total electromagnetic field. Images have been obtained on the surface of the sample $(x-y$ plane) or in a plane perpendicular to it (u-z plane). In Figs. 1(c) and 1(d) we show the near-field optical images measured in the $x-y$ plane for the two incident polarizations. For TM polarization [Fig. 1(c)], rapidly varying interference fringes are visible on the front side of the slit [the side from which the incident light impinges, $u<0$ in Fig. 1(a)]. On the other rear side $(u>0)$, a slowly varying oscillation is visible, increasing progressively when getting far away from the slit. In the case of the TE-polarized incident light [Fig. 1(d)], no fringes are observed. ${ }^{6}$

For TM polarization, two SPPs are launched and propagate in opposite directions along the metal surface, away from the slit. As the SPPs interfere with the incident and reflected plane waves, fringes with different periods appear at each side of the slit. The fringe period for $u<0$ (front side) is $\Lambda^{-}=2 \pi /\left|k_{0} \sin (\theta)+\operatorname{Re}\left(k_{\mathrm{SP}}\right)\right|$, where $k_{0}$ and $k_{\mathrm{SP}}$ are the wave-vector moduli of the incident light and of the SPP. Taking the tabulated data in Ref. 14, we get $\Lambda^{-}=493 \mathrm{~nm}$, a value in quantitative agreement with the experimental data $\left(\Lambda^{-} \approx 490 \mathrm{~nm}\right)$. For $u>0$ (rear side), there should be fringes with a period $\Lambda^{+}=2 \pi /\left|k_{0} \sin (\theta)-\operatorname{Re}\left(k_{\mathrm{SP}}\right)\right|$. Because of the highly oblique incidence, $\Lambda^{+}(\approx 21 \mu \mathrm{m})$ is much larger than the wavelength. Consequently, no fringe is observed in the finite scan window of Fig. 1(c).

To get an insight view of the field on the metal surface, we have additionally performed scans in the vertical plane $(y-z$ plane). These noncontact maps have been obtained for a small height range (from $z=0$ to $450 \mathrm{~nm}$ ) above the surface. They are shown in Fig. 2 together with calculation data obtained with a fully vectoral aperiodic Fourier modal method (a-FMM) ${ }^{15}$ In the calculation, the field is normalized such that the intensity of the incident plane wave is unitary. A good agreement is obtained between the experimental images and the calculation. Note that the scaling factor in the experiment is in arbitrary unit. For TM polarization, the bright spot near the slit corresponds to the strong field enhancement shown in the computed image. For TE polarization, the intensity of the electromagnetic field decreases right above the aperture, as previously shown in Fig. 1(d) in the $x-y$ plane. Figure 2 provides a qualitative comparison between the recorded data and the calculated ones. We next present a quantitative analysis.

The square of the total electric-field intensity, computed with the a-FMM just above the metal surface for TM polarization, is shown in Fig. 3(a) with the blue-dashed curve. We also show the experimental data with a red

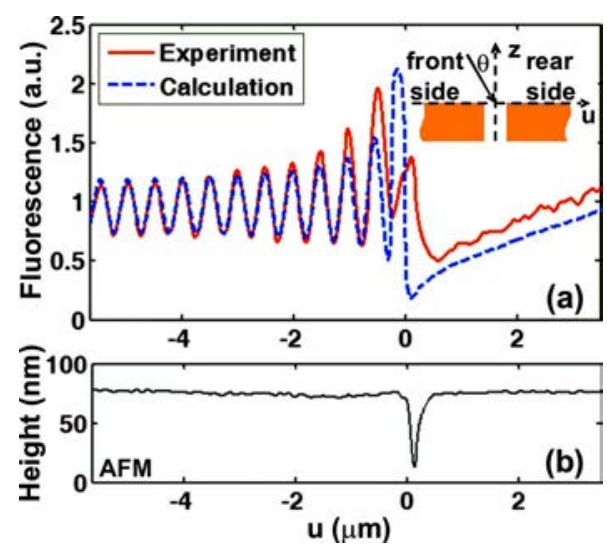

FIG. 3. (Color online) (a) Comparison between the recorded near-field data (red curve) and computational results (blue dashed curve) representing the squared intensity of the total electric field. The computed data include a spatial convolution to take into account the finite size of the fluorescent particle. (b) Atom force microscopy signal topography is recorded at the same time as the optical near-field map. The dip allows for an accurate positioning of the slit.

curve. The data are obtained from Fig. 1(c) by averaging several adjacent scans performed along the $u$-axis. The computed data $S(u)$ include a convolution of the calculated field $E_{z}(u, z)$ within the particle area $a^{2}$ to take into account the finite size $a$ of the fluorescent particle, $S(u)$ $=\int_{0}^{a} \int_{u-a / 2}^{u+a / 2}\left|E_{z}(s, z)\right|^{4} d s d z$. The electric-field component $E_{u}$ parallel to the $u$-axis is neglected in the integral since $E_{z}$ dominates in the vicinity of the metal surface. To determine the particle size $a$, we have performed an iterative optimization, minimizing the difference between $S(u)$ and the experimental data. After optimization, we obtain $a=220 \mathrm{~nm}$, a value in good agreement with the average size of our doped particles. As shown in Fig. 3(a), a quantitative agreement is observed for the fringe oscillation period and contrast between the computational and the experimental data. However, some difference also exist, especially in the immediate vicinity of the slit, $|u|<200 \mathrm{~nm}$. This deviation can be explained by considering that, as it approached the slit, the fluorescent particle penetrates inside the slit [see Fig. 3(b)] and the fluorescent signal can no longer be considered as a signature of the electric field above the surface.

To infer the SPP generation efficiency from the experimental data, we further assume that the total field is a superposition of the incident and reflected plane waves and of two SPP modes that are launched on both sides of the slit in opposite directions. Within the scope of this simplified model, the magnetic near-field is expressed as

$$
\begin{aligned}
H^{+}(u, z)= & H_{0} \exp \left(j k_{\|} u\right)\left[\exp \left(-j k_{\perp} z\right)+r \exp \left(j k_{\perp} z\right)\right] \\
& +A^{+} \exp \left(j k_{\mathrm{SP}} u\right) \exp (-\gamma z), \quad \text { for } u>0, \\
H^{-}(u, z)= & H_{0} \exp \left(j k_{\|} u\right)\left[\exp \left(-j k_{\perp} z\right)+r \exp \left(j k_{\perp} z\right)\right] \\
& +A^{-} \exp \left(-j k_{\mathrm{SP}} u\right) \exp (-\gamma z), \quad \text { for } u<0,
\end{aligned}
$$

where $\gamma=\left(k_{\mathrm{SP}}^{2}-k_{0}^{2}\right)^{1 / 2}, k_{\|}=k_{0} \sin (\theta), k_{\perp}=k_{0} \cos (\theta)$, and $H^{+}$ and $H^{-}$stand for the magnetic field for $u>0$ and $u<0$. The first terms of the right sides of Eqs. (1) and (2) represent the incident and reflected plane waves, $r$ being the Fresnel reflection coefficient of the flat interface. The second terms are related to the launched SPP modes. $H_{0}$ is a scalar denoting the amplitude of the incident plane wave, while $A^{+}$and $A^{-}$ 


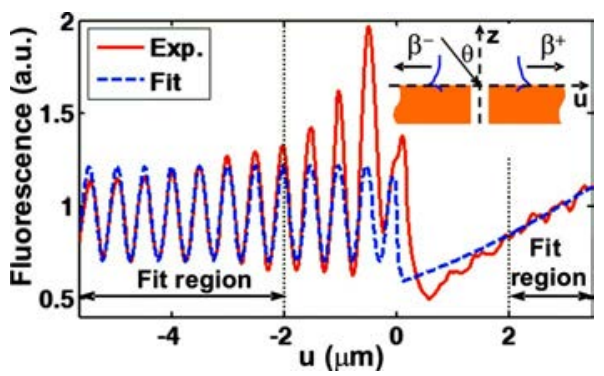

FIG. 4. (Color online) Fit of the experimental data with the model of Eqs. (3) and (5) to extract the SPP generation efficiencies. The red-solid curve corresponds to the experimental data in Fig. 3(a). The fitted curve is plotted with blue dashed curve, and the fitting region boundaries $(|u|>2 \mu \mathrm{m})$ are shown by the vertical dashed lines. In the interval $|u|<2 \mu \mathrm{m}$ (not considered for fitting), the slit near field additionally encompasses a CW that is not taken into account in the model. The inset shows the definition of SPP generation coefficients $\beta^{+}$and $\beta^{-}$.

represent the amplitudes of the SPPs launched on the rear and front sides of the slit, respectively. From Eqs. (1) and (2), the dominant $z$-component of the electric field at the metal surface $(z=0)$ is given by

$$
\begin{aligned}
E_{z}^{+}(u, z)= & -H_{0} \sin (\theta) \exp \left(j k_{\|} u\right)\left[\exp \left(-j k_{\perp} z\right)\right. \\
& \left.+r \exp \left(j k_{\perp} z\right)\right]-A^{+} k_{\mathrm{SP}} / k_{0} \exp \left(j k_{\mathrm{SP}} u\right) \\
& \times \exp (-\gamma z), \quad \text { for } u>0, \\
E_{z}^{-}(u, z)= & -H_{0} \sin (\theta) \exp \left(j k_{\|} u\right)\left[\exp \left(-j k_{\perp} z\right)\right. \\
& \left.+r \exp \left(j k_{\perp} z\right)\right]+A^{-} k_{\mathrm{SP}} / k_{0} \exp \left(-j k_{\mathrm{SP}} u\right) \\
& \times \exp (-\gamma z), \text { for } u<0 .
\end{aligned}
$$

Thus, the measured signal should be approximately given by

$$
S_{a p}(u)=\int_{0}^{a} \int_{u-a / 2}^{u+a / 2}\left|E_{z}(s, z)\right|^{4} d s d z .
$$

By using Eqs. (3)-(5), we fit the experimental data to extract the unknown parameters $H_{0}, A^{+}$, and $A^{-}$. Note that only the SPP modes are considered in the model. Actually, there are nonplasmonic quasicylindrical waves $(\mathrm{CWs})$ that are additionally scattered on the surface. As shown by recent theoretical and experimental studies, ${ }^{6,16}$ these CWs rapidly decay as one moves away from the slit. In fact, they persist over the metal surface only over a few wavelength distance. In the present case, since the incidence is highly oblique, these two additional waves (just like the SPPs) may have different strengths on both sides of the slit. Since our simplified model neglects the presence of these waves, the fit has been performed only for $|u|>2 \mu \mathrm{m}$, where the SPPs are dominant. The fitted data (blue dashed curve) are depicted in Fig. 4, together with the experimental one (red curve). A good agreement is achieved over the entire fit regions, the deviation observed for $-2 \mu \mathrm{m}<u<0 \mu \mathrm{m}$ being essentially ascribed to the presence of the $\mathrm{CW}$ on the front side of the slit. After having optimized the fitting parameters $H_{0}, A^{+}$, and $A^{-}$, one may easily derive the SPP generation efficiencies $\left|\beta^{+}\right|^{2}$ and $\left|\beta^{-}\right|^{2}$, defined as the fraction of the incident energy flow that directly impinges onto the slit entrance and that is converted into SPPs on the rear $(u>0)$ and front $(u<0)$ sides of the slit, see the inset in Fig. 4. We obtain $\left|\beta^{+}\right|^{2}=44 \%$ and $\left|\beta^{-}\right|^{2}=20 \%$. We have additionally performed calculation to check our analysis. Using the a-FMM, we have first calcu- lated the field scattered by the slit, and using the modalorthogonality formalism developed in Ref. 9, we have extracted the SPP contributions to the near-field computational data. We find $\left|\beta^{+}\right|^{2}=32.65 \%$ and $\left|\beta^{-}\right|^{2}=19.25 \%$. The second value quantitatively agrees with the experimental one, while the first value is significantly smaller. We believe that this discrepancy is due to the fact that, in contrast to the rear zone, the front zone offers several oscillation fringes in the near-field pattern and allows for an accurate fitting procedure. Finally, let us mention that both the experimental and theoretical data predict that the intensity of the SPPs launched on the rear side is larger than that launched on the front. However, as shown by other calculations, ${ }^{12}$ this is not a general property of slits or grooves.

In summary, with near-field microscopy, we have measured the field distribution in the vicinity of a nanoslit aperture in a metal film under illumination at oblique incidence. For TM polarization, the SPPs that are launched in opposite directions on the metal surface have been identified in the recorded near-field images, and with a simple model, we have extracted the SPP generation efficiency. We find a large efficiency of $20 \%$ for the SPPs that are launched at the front side of the slit, which is in fair agreement with that obtained from rigorous computational result using reciprocity arguments. A larger efficiency of $44 \%$ is also achieved at the rear side by fitting the experimental data, the reliability of which could be increased by recording more oscillation fringes. Anyway, it coincides with the theoretical prediction on the present case that the efficiency at the rear side is larger than that at the front. We believe that the present near-field approach may be of interest for characterizing the capability of various nanostructures to launch SPP on metallic surfaces.

The authors thank Michel Mortier for providing the fluorescent particles used in the near-field optical experiments.

${ }^{1}$ W. L. Barnes, A. Dereux, and T. W. Ebbesen, Nature (London) 424, 824 (2003).

${ }^{2}$ E. Ozbay, Science 311, 189 (2006).

${ }^{3}$ H. Ditlbacher, J. R. Krenn, A. Hohenau, A. Leitner, and F. R. Aussenegg, Appl. Phys. Lett. 83, 3665 (2003).

${ }^{4}$ L. Yin, V. K. Vlasko-Vlasov, A. Rydh, J. Pearson, U. Welp, S. H. Chang, S. K. Gray, G. C. Schatz, D. B. Brown, and C. W. Kimball, Appl. Phys. Lett. 85, 467 (2004).

${ }^{5}$ J. A. Sánchez-Gil and A. A. Maradudin, Appl. Phys. Lett. 86, 251106 (2005).

${ }^{6}$ L. Aigouy, P. Lalanne, J. P. Hugonin, G. Julie, V. Mathet, and M. Mortier, Phys. Rev. Lett. 98, 153902 (2007).

${ }^{7}$ F. Lopez-Tejeira, S. G. Rodrigo, L. Martín-Moreno, F. J. García-Vidal, E. Devaux, T. W. Ebbesen, J. R. Krenn, I. P. Radko, S. I. Bozhevolnyi, and M. U. Gonzalez, Nat. Phys. 3, 324 (2007).

${ }^{8}$ H. W. Kihm, K. G. Lee, D. S. Kima, J. H. Kang, and Q.-H. Park, Appl. Phys. Lett. 92, 051115 (2008).

${ }^{9}$ P. Lalanne, J. P. Hugonin, and J. C. Rodier, J. Opt. Soc. Am. A Opt. Image Sci. Vis 23, 1608 (2006)

${ }^{10}$ I. P. Radko, S. I. Bozhevolnyi, A. Boltasseva, G. Brucoli, L. MartínMoreno, and F. J. García-Vidal, Proc. SPIE 6988, 69880Q (2008).

${ }^{11}$ J. Wuenschell and H. K. Kim, IEEE Trans. Nanotechnol. 7, 229 (2008).

${ }^{12}$ H. Liu, P. Lalanne, X. Yang, and J. P. Hugonin, IEEE J. Sel. Top. Quantum Electron. 14, 1522 (2008).

${ }^{13}$ J. Gierak, A. Madouri, A. L. Biance, E. Bourhis, G. Patriarche, C. Ulysse, D. Lucot, X. Lafosse, L. Auvray, L. Bruchhaus, and R. Jede, Microelectron. Eng. 84, 779 (2007).

${ }^{14}$ E. D. Palik, Handbook of Optical Constants of Solids (Academic, New York, 1985), pp. 286-295.

${ }^{15}$ E. Silberstein, P. Lalanne, J. P. Hugonin, and Q. Cao, J. Opt. Soc. Am. A Opt. Image Sci. Vis 18, 2865 (2001).

${ }^{16}$ P. Lalanne and J. P. Hugonin, Nat. Phys. 2, 551 (2006). 